tersuchte. Der Grund, warum er das Dextrin in 2 Fällen vermisste, liegt nach Demant wahrscheinlich in der späteren Verarbeitung der betreffenden Muskeln. Diese Vermuthung scheint nicht zutreffend. Aus einer brieflichen Mittheilung Limpricht's ersehe ich nämlich, dass es sich in dem ersten Falle um ein junges, zu Schaden gekommenes Thier, in den beiden anderen Fällen um alte abgetriebene Pferde handelte, dass aber in allen $3 \mathrm{Fällen}$ das Fleisch etwa 24 Stunden post mortem in Arbeit genommen wurde. Der Grund der widersprechenden Resultate dürfte wohl lediglich in dem verschiedenen Ernährungszustande zu suchen sein.

\title{
Kommt Glycogen in der ersten Anlage des Hühnchens vor?
}

Von

\section{E. Külz.}

Die Beobachtung Bernards ${ }^{1}$ ) über das Vorkommen und die Entwickelung des Glycogens im Vogelei stammen schon aus den Jahren 1859-1863; er deponirte sie 1864 bei der Akademie in einem versiegelten Couvert, das erst 1872 eröffnet wurde. Auf das Original muss um so mehr verwiesen werden, als Bernard's Beobachtungen in den Lehrbuichern, in die sie bereits iibergegangen sind, unrichtig wiedergegeben sind. Bernard behauptet, dass das Glyeogen in der cicatricula des Hühnerei's bereits vor der Befruchtung sich findet, dass es nach der Befruchtung resp. Bebrïtung von der Cicatricula aus (in Zellen organisirt) sich im mittleren Keimblatte mit dessen Wachsthum ausbreitet. Im Original heisst es ${ }^{2}$ ): „J'ai constatè d'ailleurs, que le glycogène du blastoderme des oiseaux est de même nature chimique que le glycogène du foie et

1) Compt. rend. T. 75, 55. Bemerkt sei, dass die Arbeit Bernard's in mehreren Lehrbüchern und Jahresberichten falsch citirt ist.

2) 1. c. p. 58 . 
des organes placentaires des mammifères; sous l'influence d'agents appropriés, il se transforme en dextrine et en un sucre (glycose), qui donne par la fermentation de l'alcool de l'acide carbonique." Hiernach sollte und könnte man glauben, dass Bernard das Glycogen in Substanz dargestellt hat; man wird jedoch an dieser Meinung wieder irre, wenn man die nachfolgenden Sätze kritisch liest, aus denen hervorgeht, dass es sich nur um mikrochemische Reactionen handelt.

Weitere Zweifel an der Zuverlässigkeit resp. scharfen Begründung der Angaben tauchen auf, wenn man den folgenden Satz liest (p. 58): „De même que dans le foie et dans le placenta des mammifères, le glycogène, dans le blastoderme des oiseaux, se présente sous forme de granulations arrondies renfermées dans les cellules glycogéniques, d'une manière très-analogue à ce qui se voit pour les granules d'amidon dans les cellules végétales."

Bernard hält demnach das Glycogen in den Zellen des mittleren Keimblattes wie in den Leberzellen organisirt. Schiff $\mathbf{1}$ ) hat freilich angegeben, dass das Glycogen in den Leberzellen in Form von kleinen blassrandigen Körnchen enthalten sei, welche dicht beisanmenstehend alle Zwischenräume zwischen den grössern, dunkelrandigen, stark glänzenden Körnchen (Fett) ausfuillen, und sich durch Jod gelb bis dunkel-gelb-braun färben. H. Nasse ${ }^{2}$ ) drückt sich in dieser Beziehung sehr reservirt aus; er sah zwar nach der Fütterung mit fettloser Nahrung die Leberzellen (Hund und Kaninchen) nach Behandlang mit Jod mehr braunroth werden; allein eine blaue oder violette Färbung einzelner Körnchen in den Zellen konnte er nie wahrnehmen. Nach Bock und Hoffmann ${ }^{3}$ ), deren Beobachtungen sich auf Kaninchenleber beziehen, können die kleinen, hellen Körnchen kein Glycogen sein, denn sie waren immer vorhanden, mochte die Leber massenhaft, mochte sie kein chemisch nachweisbares Glycogen enthalten.

Was den glycogenreichen Zellen nach der Behandlung mit Jod ihre dunkle Farbe gab, erschien den genannten Autoren stets als eine amorphe, zwischen die hellen Körnchen des Zelleninhaltes

1) Arch. f. phys. Heilkunde 1857, S. 263 und M. Schiff, Untersuchungen über die Zuckerbildung in der Leber. Würzburg 1859. S. 24 u. ff.

2) Archiv des Vereins für gemeinschaftliche Arbeiten. Bd. IV. S. 97.

3) Virchow's Archiv Bd. 56. S. 201. 
eingelagerte Masse. Die Angaben von Bock und Hoffmann sind bis jetzt von Niemand geprüft worden. Ich habe reichlich Gelegenheit gehabt, auf diesen Punkt zu achten. Während ich mich von den Angaben Schiff's nicht überzeugen konnte, muss ich die Richtigkeit der Beobachtungen von Bock und Hoffmann in allen Punkten bestätigen. Vorgerïektere Sehüler, denen ich die Prüfung jener Angaben zur Aufgabe stellte, sind zu ganz demselben Resultate gekommen. Ich benutze die Gelegenheit, auf die Angaben von Bock und Hoffmann um so nachdricklicher binzuweisen, als sie noch nicht die gebührende Berücksichtigung gefunden haben und Bernard in seinen "Vorlesungen uber den Diabetes ${ }^{2}$ " seine Ansicht festbält.

Wenn nun die Behauptung von der Organisation des GIycogens in den Zellen der Leber, deren Glycogengehalt Niemand bestreitet, sich als irrig oder mindestens als höchst zweifelhaft herausgestellt hat, so muss man die Angaben Bernard's ${ }^{3}$ ) über die Organisation des Glycogens in der ersten Anlage des Hiuhnerembryos, aus der noch Niemand bis jetzt das Glyeogen in Substanz dargestellt hat, mit um so grösserer Vorsicht aufnehmen; man darf sie nicht ohne weitere Prüfung als Thatsachen der Physiologie einreihen. Der mikroskopische Nachweis des Glycogens ist durchaus trügerisch und kann für sich allein nichts entscheiden. Ioh will damit nicht behaupten, dass der mikrochemische Nachweis des Glycogens bei einer Untersuchung nicht leitend sein könne.

Für mich handelt es sich zunächst darum: „Kommt Glycogen iiberhaupt in der ersten Anlage des Hiubnchens vor, kann man es in Substanz daraus darstellen?

Zur Entscheidung dieser Frage liess ich 200 Hühnereier bis zur Leberanlage ${ }^{4}$ ) (circa 60 Stunden) bebrüten. 116 Eier waren angegangen. Die Embryonen wurden frisch in siedendes Wasser geworfen, nochmals zerkleinert und ausgiebig gekocht. Mit Hülfe

1) Vergl. auch Tscherinow in Virchow's Archiv Bd. 47. S. 110.

2) Uebersetzung von Posner S. 326. Anmerkung 2.

3) Ueber die Art der Organisation des Leberglycogens hat sich Bernard meines Wissens nirgends speciell geäussert.

4) Wenn auch Bernard (Uebersetzung von Posner S. 325) angiebt, dass die Leber im Beginn ihrer Entwickelung kein Glycogen enthäl, so glaubte ich der Sicherheit der Schlüsse halber ïber diese Zeit nicht hinausgehen zu dürfen. 
E. Kulz:

der Brücke'schen Methode konnte ich schliesslich noch nicht 1 Centigramm eines weissen Pulvers abscheiden, das folgende Eigenschaften zeigte: es löste sich in kaltem Wasser mit Opalescenz, wurde daraus durch Alcohol gefällt. Unter Zusatz eines Jodsplitters röthete sich die wässerige Lösung, die rothe Farbe verschwand bei dem Erhitzen, um nach dem Frkalten wiederzukehren. Die eine Hälfte der wässerigen Lösung wurde mit HCl gekocht, die andere mit frischem reinen Parotidenspeichel versetzt. Beide Proben reducirten nach Trommer behandelt deutlich. Ich halte hiermit das Vorkommen von Glycogen in der ersten Anlage des Hühnchens für bewiesen.

Dank dem freundlichen Entgegenkommen der Herren Fabrikanten Trapp und Münch in Friedberg wurde es mir möglich, von 5000 frischen Hühnereiern die cicatriculae $\mathrm{zu}$ isoliren. Leider ist es mir nicht gelungen, auch nur eine Spur Glycogen daraus zu gewinnen.

\section{Bildet der Muskel selbständig Glycogen?}

Von

E. Intr.

Die experimentelle Entscheidung der hier aufgeworfenen Frage ist bis jetzt noch nicht versucht worden. Folgende Thatsachen machen es nicht unwahrscheinlich, dass auch der Muskel selbstständig Glyeogen bilden kann:

1. Das verschiedene Verhalten von Leber- und Muskelglycogen ggegen Jod').

1) Auf diesen Unterschied hat Naunyn (Arch. f. experim. Pathol. u. Pharmak. 3, 97) bei Hühnern, wo er besonders auffällig und leicht zu constatiren ist, zuerst aufmerksam gemacht. Nach ihm giebt das Leberglycogen mit Jod eine braunrothe, das Muskelglycogen eine „prachtvoll violette bung. Naunyn will übrigens mit Recht vorläufig die Erfahrungen an Hühnern über das Muskelglycogen nicht ohne weiteres verallgexaeinert wissen. 